\title{
Catalogue général de vitesses radiales moyennes pour les étoiles galactiques $^{\star}$
}

\section{Mean radial velocities catalog of galactic stars}

\author{
M. Barbier-Brossat et P. Figon \\ Observatoire de Marseille, 2 place Le Verrier, F-13248 Marseille Cedex 04, France
}

Reçu le 4 novembre ; accepté le 24 novembre 1999

\begin{abstract}
We present a catalogue of mean radial velocities for Galactic stars which supplements the catalogue WEB (Duflot et al. 1995) with observations published through december 1990. The catalogue contains new mean velocities for 20574 stars. Only observations obtained with adequate spectral resolution and standardized to the IAU or Wilson (1953) velocity systems have been used. Entries in the WEB mean radial velocities catalogue have been taken to represent the mean of all earlier measurements, to which the new data have been combined. The sources of new data are referenced. To make the work of users easier, we have included in our catalogue the results of the catalogue WEB, with agreement of the author.
\end{abstract}

Key words: catalogs — stars: general — stars: kinematics

\section{Introduction}

Ce catalogue est l'aboutissement d'un travail commencé en 1976 à la demande de la commission 30 de l'UAI. Un premier catalogue de vitesses radiales moyennes, qui prenait en compte les vitesses radiales publiées jusqu'en 1980, est paru en 1989 (Barbier-Brossat 1989). Ce second catalogue comprend les vitesses publiées jusqu'en 1990 ; il donne une vitesse moyenne nouvelle pour 20546 étoiles de notre galaxie. Ce sera le dernier catalogue de vitesses moyennes publié par l'Observatoire de Marseille. Ce travail a nécessité la réalisation d'une bibliographie des vitesses radiales publiées depuis 1970, date à laquelle s'arrête le catalogue bibliographique de Abt \& Biggs (1972). Trois catalogues bibliographiques ont été successivement publiés (Barbier-Brossat \& Petit 1986, 1990;

Send offprint requests to: M. Barbier-Brossat

* Tables 1 and 2 are only available in electronic form at the CDS via anonymous ftp to cdsarc.u-strasbg.fr (130.79.128.5).
Barbier-Brossat et al. 1994), le dernier réunissant toutes les vitesses radiales publiées depuis 1970 jusqu'à 1990 inclus. Nous avons joint à nos résultats les vitesses moyennes du catalogue WEB (Duflot et al. 1995), de façon à ce que l'utilisateur puisse disposer, dans une seule publication, de tous les résultats de vitesses moyennes disponibles jusqu'en 1990 ; il comprend ainsi les vitesses moyennes pour 36145 étoiles. Cela a été effectué en accord avec Mme M. Duflot que nous remercions vivement.

\section{Origine des vitesses radiales utilisées}

- Pour les vitesses publiées antérieurement à 1970.

Il était difficile de revenir pour chaque étoile aux vitesses d'origine. Nous avons utilisé les vitesses moyennes données dans le catalogue WEB (Duflot et al. 1995). Ce catalogue reprend, outre le catalogue d'éléments orbitaux de doubles spectroscopiques de Batten et al. (1989), les catalogues de vitesses moyennes de Wilson (1953) et d'Evans (1970). Il regroupe donc les vitesses radiales moyennes des étoiles dont les vitesses radiales ont été publiées avant 1970. Le WEB a apporté plusieurs améliorations aux trois catalogues précédents : les identificateurs donnés permettent le plus souvent l'accès à la base de données SIMBAD du Centre de Données astronomiques de Strasbourg ; la plupart des problèmes d'identification posés par les étoiles faibles, les étoiles d'amas ou les étoiles doubles ont été résolus ; des erreurs relevées dans ces catalogues, comme dans tout catalogue, ont été corrigées.

Des problèmes se sont posés pour établir la jonction avec le catalogue d'Evans. D'une part, ce catalogue n'a pas été terminé : il y manque les trois dernières heures. Nous avons utilisé, pour combler cette lacune, le catalogue bibliographique de Abt \& Biggs, en reprenant pour $21 \mathrm{~h}$, 22 h et 23 h la bibliographie à partir de 1952, date à laquelle s'arrête le catalogue de Wilson.

D'autre part, les dates auxquelles Evans a arrêté sa bibliographie ne sont pas les mêmes pour toutes les heures. 
Des difficultés se sont donc présentées pour les années de 1968 à 1970 : c'est ainsi, que pour une même publication, les vitesses des étoiles situées aux dernières heures peuvent être incluses dans le catalogue, alors que celles situées aux premières heures ne le sont pas. Nous avons essayé de résoudre ce problème cas par cas. En particulier, la publication de Heard (1956) n'a été prise en compte que partiellement par Evans : pour les étoiles situées à $15 \mathrm{~h}$ d'ascension droite et pour celles situées de 18 à $20 \mathrm{~h}$. Nous avons introduit dans notre catalogue toutes les vitesses radiales de cette publication non prises en compte par Evans.

Enfin, Evans a utilisé des résultats fournis par Hube avant leur publication, ceci pour les étoiles d'ascension droite comprises entre 0 h et $11 \mathrm{~h}$. De nouvelles vitesses ont été mesurées ensuite par l'auteur pour ces étoiles, d'autres supprimées, des étoiles ajoutées. Les vitesses moyennes publiées ensuite par Hube (Hube 1970) pour ces étoiles ne correspondent pas à celles qui ont été utilisées par Evans dans son catalogue. Nous avons introduit les étoiles manquantes et rendu les vitesses des autres conformes à la publication, y compris dans le catalogue WEB.

Nous avons également, dans notre catalogue, remplacé les vitesses radiales obtenues à l'aide du petit prisme objectif et publiées durant les années 1955-1968 par la réévaluation qui en a été faite par les auteurs (Fehrenbach et al. 1996).

Enfin, les vitesses radiales obtenues par Fehrenbach et son équipe (Fehrenbach et al. 1987) à l'aide du spectrographe Marly ont été améliorées par la mesure de nouveaux clichés obtenus au même spectrographe (Grenier et al. 1999). Nous avons donc introduit ces nouveaux résultats à la place des anciens, mais nous n'avons pas pris en compte les résultats concernant les étoiles ne figurant pas dans la première publication.

\section{- Pour les vitesses radiales publiées après 1970.}

Nous avons utilisé la bibliographie de notre dernier catalogue bibliographique (Barbier-Brossat et al. 1994). Il est particulièrement important que les vitesses utilisées pour le calcul de la vitesse moyenne d'une étoile soient bien raccordées à un même système de vitesses radiales. Pour cela, nous n'avons retenu que les publications dont les résultats sont bien raccrochés au système d'étoiles standards de l'UAI $(1955,1973)$ ou aux vitesses de bonne qualité du catalogue de Wilson : ces dernières ont été utilisées par de nombreux auteurs, en particulier pour les étoiles de type spectral OBA, pour lesquelles n'existent pas d'étoiles standards ; le système de vitesses moyennes du catalogue de Wilson est, en effet, très proche du point zéro (Evans 1967).

Pour toutes les publications utilisées dans notre catalogue et comprenant un nombre suffisant d'étoiles, nous avons comparé entre elles les vitesses radiales des étoiles communes aux différentes listes. Nous avons également comparé, pour ces publications, les vitesses des étoiles avec celles qu'elles avaient en commun avec le catalogue WEB et les listes d'étoiles standards de l'UAI ; nous n'avons pas observé de différence systématique suffisamment importante et bien définie, en fonction du nombre d'étoiles communes et de leurs types spectraux, pour nécessiter une correction, sauf pour une publication (Bakos 1974). Cet article concerne des étoiles doubles visuelles de type spectral F, G, K ou M dont 51 ont des vitesses radiales de qualité a ou b dans le catalogue Wilson. Pour ces 51 étoiles, la moyenne des différences entre les vitesses radiales de Bakos et celles de Wilson est de $-3,2 \mathrm{~km} \mathrm{~s}^{-1}$. Nous avons donc ajouté $+3 \mathrm{~km} \mathrm{~s}^{-1}$ à toutes les vitesses de l'article de Bakos.

Selon les recommandations de l'UAI (UAI 1973), nous avons également effectué une correction de $-3 \mathrm{~km} \mathrm{~s}^{-1}$ sur les vitesses provenant d'une publication de Heard (Heard 1956).

\section{Calcul des vitesses moyennes}

Nous avons éliminé toutes les vitesses radiales obtenues avec une dispersion inférieure à $100 \AA / \mathrm{mm}$ ou pour lesquelles l'auteur ne donne aucune indication sur la dispersion ou la résolution.

Nous avons, bien sûr, éliminé toutes les vitesses déjà publiées dans un article précédent. Lorsqu'un auteur fait la moyenne de ses résultats avec des vitesses précédemment utilisées dans le calcul des vitesses moyennes du WEB, nous n'avons pris en compte que les nouveaux résultats.

Pour les vitesses radiales obtenues à l'aide du petit prisme-objectif Fehrenbach, nous n'avons utilisé que les résultats provenant d'au moins 3 mesures. Quant aux vitesses moyennes du WEB et à celles publiées par les auteurs, nous les avons traitées comme une somme de $n$ vitesses, $n$ étant le nombre de vitesses ayant servi pour calculer la moyenne, toutes égales à la vitesse moyenne donnée.

Nous avons établi une pondération entre les différentes vitesses d'une même étoile, qui tient compte à la fois de la technique et de la dispersion utilisées par l'auteur. Si l'auteur publie une vitesse provenant de plusieurs mesures obtenues avec des dispersions différentes, nous prenons, pour la vitesse, la dispersion la plus faible.

Pondération adoptée :

- poids 2 : - vitesses radiales photoélectriques par corrélation croisée ;

- spectrographe à fente, dispersion $<40 \AA / \mathrm{mm}$;

- poids 1 : - tube-image ;

- spectrographe à fente, dispersion $40 \leq d \leq 100 \AA / \mathrm{mm}$.

Si les vitesses auxquelles on donne un certain poids résultent de mesures très précises et bien raccordées, il est 
évident qu'une pondération, quelle qu'elle soit, ne peut qu'améliorer la vitesse moyenne de l'étoile, si cette dernière a une vitesse constante. Mais, lorsque nous effectuons le calcul d'une vitesse moyenne pour une étoile variable, les vitesses utilisées ne sont en général pas réparties également sur la courbe de variation. En pondérant, nous favorisons certains résultats par rapport à d'autres, et la moyenne est alors modifiée d'une valeur qui dépend de la répartition des différents résultats et de l'écart de vitesse entre le maximum et le minimum de vitesse de la variable. Cette valeur est tout à fait aléatoire et, dans certains cas, peut être importante.

Nous avons étudié les conséquences de notre pondération sur les vitesses moyennes de notre catalogue. Nous n'avons pris en compte que les étoiles pour lesquelles nous disposions de 3 mesures au moins. Nous avons calculé la vitesse moyenne, une première fois avec pondération, une autre sans pondération. Si l'on compare les vitesses moyennes obtenues ainsi, environ $20 \%$ des étoiles voient leur vitesse moyenne modifiée. Ce pourcentage est faible, car notre bibliographie s'arrête en 1990 et, à cette époque, il y avait encore peu de vitesses suffisamment précises pour leur affecter un poids.

Pour les étoiles qui ont un sigma inférieur à $3 \mathrm{~km} \mathrm{~s}^{-1}$, ce qui représente à peu près $50 \%$ des étoiles prises en compte, la différence de vitesse est en moyenne de $\pm 0,4 \mathrm{~km} \mathrm{~s}^{-1}$. Plus le sigma augmente, plus cette différence de vitesses devient importante. Elle est déjà de $\pm 1,5 \mathrm{~km} \mathrm{~s}^{-1}$ pour un sigma compris entre 3 et $6 \mathrm{~km} \mathrm{~s}^{-1}$ et atteint $\pm 6 \mathrm{~km} \mathrm{~s}^{-1}$ pour les sigmas supérieurs à $6 \mathrm{~km} \mathrm{~s}^{-1}$. On remarque que la pondération a également comme effet secondaire de diminuer le sigma et de masquer ainsi la variabilité de certaines étoiles.

Si l'on doit, plus tard, faire un nouveau catalogue de vitesses moyennes, il faudra prendre en compte les effets de la pondération et ne pas affecter un poids trop fort aux meilleures mesures.

Pour les étoiles doubles, lorsqu'il s'agit de la vitesse systémique, elle est suivie du nombre de mesures ayant été utilisées pour son calcul, ainsi que de ORB. Lorsqu'il y a plusieurs publications, nous publions la dernière en date.

Pour les étoiles Céphéides ou RR Lyrae, nous publions la vitesse qui nous semble la meilleure, compte-tenu de leur variabilité ; elle est suivie de CEPH ou RRLY.

Pour les étoiles standards de l'UAI, nous publions la vitesse recommandée par l'UAI.

\section{Précision des vitesses moyennes}

Nous donnons séparément le nombre de mesures et le nombre de publications ayant été utilisées pour calculer la vitesse moyenne, ainsi que l'erreur standard, ce dernier critère étant important pour la mise en évidence de la variabilité d'une étoile.
L'erreur standard est calculée suivant la formule :

$$
\sigma=\left[\frac{\left(n(n-1) \sigma_{1}^{2}+m(m-1) \sigma_{2}^{2}\right.}{(n+m)(n+m-1)}+\frac{\left(n m\left(V_{1}-V_{2}\right)^{2}\right.}{(n+m)^{2}(n+m-1)}\right]^{1 / 2}
$$

- $V_{1}, V_{2}=$ vitesses publiées par les auteurs et qui contribuent au calcul de la vitesse moyenne;

- $n, m=$ nombre de mesures utilisées pour le calcul de $V_{1}$ et $V_{2}$;

- $\sigma_{1}, \sigma_{2}=$ erreurs standards correspondant à $V_{1}$ et $V_{2}$.

Tableau 3. Qualités données aux vitesses radiales obtenues à l'aide du petit prisme-objectif Fehrenbach, $p$ étant l'écart probable

A
B $2,5<p \leq 2,5 \mathrm{~km} \mathrm{~s}^{-1}$
$\mathrm{C} \quad 5,0<p \leq 10,0$
$\mathrm{D} 10,0 \leq p$

Lorsque le nombre de vitesses contribuant au calcul de la vitesse moyenne est supérieur à deux, nous avons procédé par calculs successifs.

Si l'auteur publie une moyenne provenant de plusieurs résultats et ne donne pas l'erreur standard, nous l'avons calculée, à partir des valeurs individuelles des vitesses. Lorsque ce n'était pas possible, nous avons considéré la vitesse publiée comme provenant d'une seule mesure.

Pour les qualités des vitesses obtenues à partir du petit prisme-objectif Fehrenbach, nous avons tenu compte des définitions de ces qualités données par Duflot \& Fehrenbach (1956a,b) et calculé l'erreur standard correspondante.

Dans le catalogue WEB, la qualité d'un résultat est donnée par une lettre attribuée à la vitesse moyenne suivant certains critères, légèrement différents pour chaque auteur.

Pour les vitesses provenant du catalogue WEB, nous avons établi une correspondance entre les qualités A, B, C, D, E et l'erreur standard, suivant les définitions de ces qualités données par les auteurs. Nous avons éliminé les vitesses de qualité E lorsqu'il n'y avait qu'une seule mesure, car elles sont de mauvaise qualité, soit parce que le spectre était sous-exposé, soit parce que les raies étaient très larges ou trop peu nombreuses. Evans n'ayant pas déterminé les qualités des vitesses moyennes entre $17 \mathrm{~h} 11 \mathrm{~min}$ et $18 \mathrm{~h}$, nous avons calculé l'erreur standard à partir des vitesses utilisées pour obtenir la moyenne.

\section{Informations présentes dans le Catalogue}

Champ 1 : Numéro BF suivi d'une * lorsque l'étoile provient du catalogue WEB. 
Tableau 4. Correspondance entre les lettres définissant les qualités des vitesses et les erreurs-standards adoptées, pour les catalogues de Wilson, d'Evans et pour les vitesses obtenues au prisme-objectif Fehrenbach (Duflot et al. 1956a,b)

$\begin{array}{cc}\text { qualité } & \text { erreur standard } \\ \text { Wilson, Evans } & \text { adoptée } \\ \text { a } & 0,74 \\ \text { b } & 1,78 \\ \text { c } & 3,70 \\ \text { d } & 7,40 \\ \text { e } & 10.0\end{array}$

$\begin{array}{cc}\text { qualité } & \text { erreur standard } \\ \text { Duflot, Fehrenbach } & \text { adoptée } \\ \text { A } & \frac{2.5}{0.8}\left(\frac{n}{n-1}\right)^{1 / 2} \\ \text { B } & \frac{5.0}{0.8}\left(\frac{n}{n-1}\right)^{1 / 2} \\ \text { C } & \frac{10.0}{0.8}\left(\frac{n}{n-1}\right)^{1 / 2} \\ \text { D } & \frac{25.0}{0.8}\left(\frac{n}{n-1}\right)^{1 / 2}\end{array}$

Champ 2 : Identification de l'étoile dans les catalogues HD ou HDE. Une lettre A, B, C etc. indique qu'il s'agit d'une des composantes de l'étoile, ce qui est d'ailleurs confirmé par son numéro dans le catalogue CCDM lorsqu'elle y figure. Cette lettre n'est mise que pour éviter la confusion avec une autre composante de la même étoile quand on effectue les moyennes. Une lettre $\mathrm{i}$ ou $\mathrm{j}$ indique qu'il s'agit de composantes d'une binaire spectroscopique. Le signe / signifie que la moyenne concerne 2 étoiles ayant des numéros HD qui se suivent ou que la vitesse radiale moyenne est relative à la mesure simultanée des 2 composantes d'une même étoile.

Champ 3 : Identification de l'étoile dans les catalogues Bonner Durchmusterung, Cordoba Durchmusterung et Cape Photographic Durchmusterung, respectivement BD, CD, CPD.

Le catalogue $\mathrm{BD}$ pour les déclinaisons de +90 degrés à -22 degrés,

Le catalogue $\mathrm{CD}$ pour les déclinaisons de -23 degrés à -51 degrés,

Le catalogue CPD pour les déclinaisons de -52 degrés à -90 degrés,

selon la convention adoptée par le HD. Il peut arriver que, dans certaines de ces zones, l'étoile ne figure pas dans le catalogue correspondant ou qu'elle ne soit pas incluse dans Simbad, ce qui explique sa désignation dans un autre catalogue.

Les lettres et signes pouvant éventuellement figurer après l'identificateur ont la même signification que pour les numéros HD.

Lorsque nous avons nous-mêmes établi la correspondance entre ces catalogues et l'identification donnée par l'auteur, ces numéros sont suivis du symbole ":".

Champ 4: Autres identifications : nous avons essayé de conserver les identificateurs donnés par les auteurs tout en respectant, chaque fois que cela était possible, les appellations recommandées par "The First Dictionary of the Nomenclature of Celestial Objects" (Fernandez et al. 1983) et "The Second Dictionary..." (Lortet \& Spite 1986).

Pour les étoiles n'ayant pas été référencées dans un catalogue ou n'ayant pas de désignation propre, nous donnons le numéro d'ordre dans la publication référencée en dernière colonne, précédé des initiales de l'auteur.

Champ 5 : Numéro du catalogue CCDM (Dommanget \& Nys 1994). Le numéro CCDM est suivi d'une lettre correspondant à la composante visuelle observée. Dans le cas de composantes très proches, nous avons utilisé le code utilisé par Mme M. Duflot dans le catalogue WEB, sauf lorsque l'auteur signalait expressément la composante étudiée.

Champ 6 : Numéro HIP du catalogue d'entrée HIPPARCOS, (Turon et al. 1992).

Champ 7 : Numéro du catalogue WEB (Duflot et al. 1995).

Champ 8 : Ascension droite et déclinaison pour l'équinoxe 2000, provenant de la base Simbad au printemps 1998. Lorsque les coordonnées de l'étoile ne figuraient pas dans Simbad, nous les avons précessées nous-mêmes à partir des coordonnées 1950 de notre dernier catalogue bibliographique ; elles sont alors suivies d'une arobasque (@). Les entrées se font par ascension droite croissante et déclinaison décroissante.

Un astérisque dans la colonne des dixièmes de minute d'ascension droite indique que les coordonnées sont approximatives. En particulier pour certaines étoiles d'amas dont les coordonnées ne sont pas connues, nous donnons les coordonnées du centre de l'amas, suivies d'un astérisque dans la colonne des dixièmes de minute.

Champ 9 : Magnitudes visuelles ou photoélectriques : ce sont celles données par Simbad, sauf lorsqu'elles ne figurent pas dans la base ou lorsque celles données par l'auteur sont plus précises. La magnitude précédée d'un astérisque est soit une magnitude $B$, soit une magnitude photographique.

Champ 10 : Types spectraux : ce sont ceux donnés par Simbad. Nous avons conservé ceux publiés par les auteurs, s'ils ne figurent pas dans la base ou s'ils sont plus précis que dans Simbad, en particulier en ce qui concerne la classe de luminosité. 
Champ 11 : Vitesse radiale moyenne : elle est publiée sans décimale lorsque l'erreur standard moyenne est supérieure à 3,70 .

Pour les étoiles doubles spectroscopiques, nous publions la vitesse systémique de la dernière orbite publiée depuis 1987, date à laquelle se termine le catalogue de Batten (Batten et al. 1989).

Pour les étoiles doubles ou multiples, nous publions la dernière orbite publiée.

Pour les étoiles RR Lyrae et les Céphéides, nous publions la vitesse $V_{0}$ donnée par les auteurs qui nous semble la meilleure.

Pour les étoiles standards de l'UAI, nous publions les vitesses recommandées par l'UAI, (UAI 1955, UAI 1973). Pour l'étoile HD 14969, nous avons suivi les recommandations de Griffin (Griffin 1980) et l'avons ôtée de la liste des étoiles de vitesse radiale standard.

Champ 12 : Erreur standard moyenne ou qualité du Web (A, B, C, D, E) s'il y a une * dans le champ 1.

ORB = l'étoile a une orbite dont nous donnons la dernière vitesse publiée ;

RRLY = l'étoile est une RR Lyrae ;

$\mathrm{CEPH}=$ l'étoile est une céphéide ;

STND = l'étoile est une standard de l'UAI.

Champ 13 : Nombre de mesures ayant été utilisées pour le calcul de la vitesse moyenne.

Champ 14: Nombre de références utilisées pour le calcul de la vitesse moyenne, y compris celle du catalogue WEB, lorsqu'elle existe.

Champ 15 : Références bibliographiques.

Elles mentionnent : le nom de la revue, suivant l'abréviation publiée dans le Tableau 2, le numéro du volume, la page et l'année.

Pour une même étoile, les entrées sont données par années croissantes.

Dans le cas des IBVS, seuls figurent le numéro de la publication et l'année, aucune pagination n'étant indiquée sur les fascicules.

Remerciements. Nous sommes heureux d'exprimer nos très vifs remerciements :

- Aux membres de la Commission 30 de l'UAI, et plus particulièrement aux membres du Groupe de Travail qui nous ont apporté approbations et critiques constructives.

- À Mme M. Duflot dont la compétence était toujours disponible, avec qui nous avons eu de fréquentes et très profitables discussions, qui nous a beaucoup aidés lors de l'introduction dans notre catalogue des numéros des catalogues Web, Hipparcos et CCDM et qui a eu le mérite de faire une relecture complète de cet ouvrage.
Nous adressons également nos remerciements à toute l'équipe du Centre de Données astronomiques de Strasbourg pour tous les échanges fructueux que nous avons eus lors de l'identification croisée de notre catalogue avec la base Simbad.

Ce catalogue est disponible au Centre de Données astronomiques de Strasbourg. L'observatoire de Marseille en éditera une version imprimée en fonction de la demande.

\section{Bibliographie}

Abt H.A., Biggs E.S., 1972, Bibliography of stellar radial velocities. Latham Process Corp., New York

Bakos G.A., 1974, AJ 79, 866

Barbier-Brossat M., Petit M., 1986, A\&AS 65, 59

Barbier-Brossat M., 1989, A\&AS 80, 67

Barbier-Brossat M., Petit M., 1990, A\&AS 85, 885

Barbier-Brossat M., Figon P., Petit M., 1994, A\&AS 108, 603

Batten A.H., Fletcher J.M., Mac Carthy D.G., 1989, Pub. Dominion Astroph. Observ. Vol. XVII, 1-317

Beavers W.I., Eitter J.J., 1986, ApJS 62, 147

Dommanget J., Nys O., 1994, Com. de l'Obs. Royal de Belgique, Série A, No. 115

Duflot M., Figon P., Meyssonnier N., 1995, A\&AS 114, 269

Duflot M., Fehrenbach Ch., 1956a, Publ. Obs. Haute-Provence III, No. 41

Duflot M., Fehrenbach Ch., 1956b, Publ. Obs. Haute-Provence III, No. 49

Evans D.S., 1967, IAU Symp. No. 30, Determination of radial velocities and their applications, Batten A.H. and Heard J.E. (eds.). Academic Press London and New-York, p. 57

Evans D.S., 1970, General catalogue of stellar radial velocities, en communication privée, édité et distribué par l'observatoire de Marseille, avec accord de l'auteur

Fehrenbach Ch., Burnage R., Duflot M., et al., 1987, A\&AS 71,263

Fehrenbach Ch., Duflot M., Genty V., Amieux G., 1996, Bulletin d'information du Centre de Données Astronomiques de Strasbourg, No. 48

Fernandez A., Lortet M.C., Spite F., 1983, A\&AS 52, 4, 1.17.14

Grenier S., Baylac M.O., Rolland L., et al., 1999, A\&AS 137, 451

Griffin R.F., 1980, MNRAS 190, 711

Heard J.F., 1956, David Dunly Obs. 2, 107

Hube D.P., 1970, Mem. Roy. Astron. Soc. 72, 233

Lortet M.C., Spite F., 1986, A\&AS 64, 329

Lortet M.C., Borde S., Ochsenbein F., 1994, A\&AS 107, 193

Transactions of the International Astronomical Union, Reports 1955, Vol. IX, Commission 30

Transactions of the International Astronomical Union, Reports 1973, Vol. XV A, Commission 30

Turon C., et al., 1992, The HIPPARCOS Input Catalogue, ESA SP-1136

Wilson R.E., 1953, General Catalogue of Stellar Radial Velocities, Carnegie Inst. of Washington Publ. 601, Washington DC 
Tableau 5. Tableau des correspondances

$\begin{array}{ll}\text { A\&A } & \text { Astronomy and Astrophysics } \\ \text { A\&AS } & \text { Astronomy and Astrophysics Supplement Series } \\ \text { AFA } & \text { Arkiv für Astrophysik } \\ \text { AJ } & \text { Astronomical Journal } \\ \text { AN } & \text { Astronomische Nachrichten } \\ \text { AP } & \text { Astrophysics (URSS) } \\ \text { ApJ } & \text { Astrophysical Journal } \\ \text { ApJS } & \text { Astrophysical Journal Supplement Series } \\ \text { ASS } & \text { Astrophysics and Space Science } \\ \text { AT } & \text { Annals of the Tokyo Astronomical Observatory } \\ \text { BAIC } & \text { Bulletin of the Astronomical Institutes of Czechoslovakia } \\ \text { BCDS } & \text { Bulletin du Centre de Données Stellaires de Strasbourg } \\ \text { DAO } & \text { Publications of the Dominion Astrophysical Observatory } \\ \text { IBVS } & \text { Information Bulletin on Variable Stars } \\ \text { JAA } & \text { Journal of Astrophysics and Astronomy (Inde) } \\ \text { MAKL } & \text { Mededelingen van Het Astronomich Institut van de Katholieke Universiteit Leuven } \\ \text { MEM } & \text { Memoirs of the Royal Astronomical Society } \\ \text { MN } & \text { Monthly Notices of the Royal Astronomical Society } \\ \text { MSAI } & \text { Memorie della Società Astronomica Italiana } \\ \text { OBS } & \text { The Observatory } \\ \text { PASJ } & \text { Publications of the Astronomical Society of Japan } \\ \text { PASP } & \text { Publications of the Astronomical Society of Pacific } \\ \text { PDDO } & \text { Publications of the David Dunlap Observatory } \\ \text { POAS } & \text { Publications de l'Observatoire Astronomique de Strasbourg } \\ \text { POHP } & \text { Publications de l'Observatoire de Haute-Provence } \\ \text { RASC } & \text { Journal of the Royal Astronomical Society of Canada } \\ \text { RGOA } & \text { Royal Greenwich Observatory Annals } \\ \text { RMAA } & \text { Revista Mexicana de Astronomia y Astrofisica } \\ \text { ROB } & \text { Royal Observatory Bulletin } \\ \text { SAL } & \text { Soviet Astronomy - Letters } \\ \text { SAOC } & \text { South African Astronomical Observatory Circulars } \\ \text { UAI } & \text { Transactions of the International Astronomical Union } \\ & \end{array}$

Erreurs

Des erreurs, trouvées dans les différentes publications lors du relevé des vitesses radiales pour notre catalogue bibliographique, ont été signalées dans celui-ci. Quelques-unes ont encore été relevées en réalisant ce catalogue. Nous les donnons ci-dessous :

$\begin{array}{lll}\text { AAP 13, 447-1971 } & \text { HD 27139 } & \text { lire HD 27149 } \\ \text { AAP 118, 255-1983 } & \text { V539 ARA, }-55 \text { deg.35 } & \text { lire }-53 \text { deg.35 } \\ \text { AAP 118, 255-1983 } & \text { ZET PHE } \gamma=-15,3 & \text { lire } \gamma=+15,3 \\ \text { AAP 219,125-1989 } & \text { HD 73558 } & \text { lire HD 73598 } \\ \text { AAP 236, 409-1990 } & \text { HD 214860 } & \text { lire HD 214680 } \\ \text { AAPS 61, 303-1985 } & \text { HD 6085 } & \text { lire HD 6805 } \\ \text { AJ 81, 364-1976 } & \text { anonyme } & \text { lire C* 590 } \\ \text { AJ 81, 364-1976 } & \text { anonyme } & \text { lire C* 791 } \\ \text { AJ 96, 719-1988 } & \text { GL 378.1 } & \text { lire GL 378.2 } \\ \text { APJS 62, 147-1986 } & \text { HR 6134= HD 148474 } & \text { lire HD 148478 } \\ \text { APJS 73, 841-1990 } & \text { C*2866 est Cya 51 et non Cya 50 } & \\ \text { PASP 86, 742-1974 } & \text { HD 4967 VR=116 } & \text { lire VR=11,6 } \\ \text { PDDO 2, 107-1956 } & \text { HD 208277 } & \text { lire HD 208276 } \\ \text { RGOA 14, 1-1981 } & \text { AV SER 15h18,8(1900) } & \text { lire 15h58,8 }\end{array}$


Principaux identificateurs :

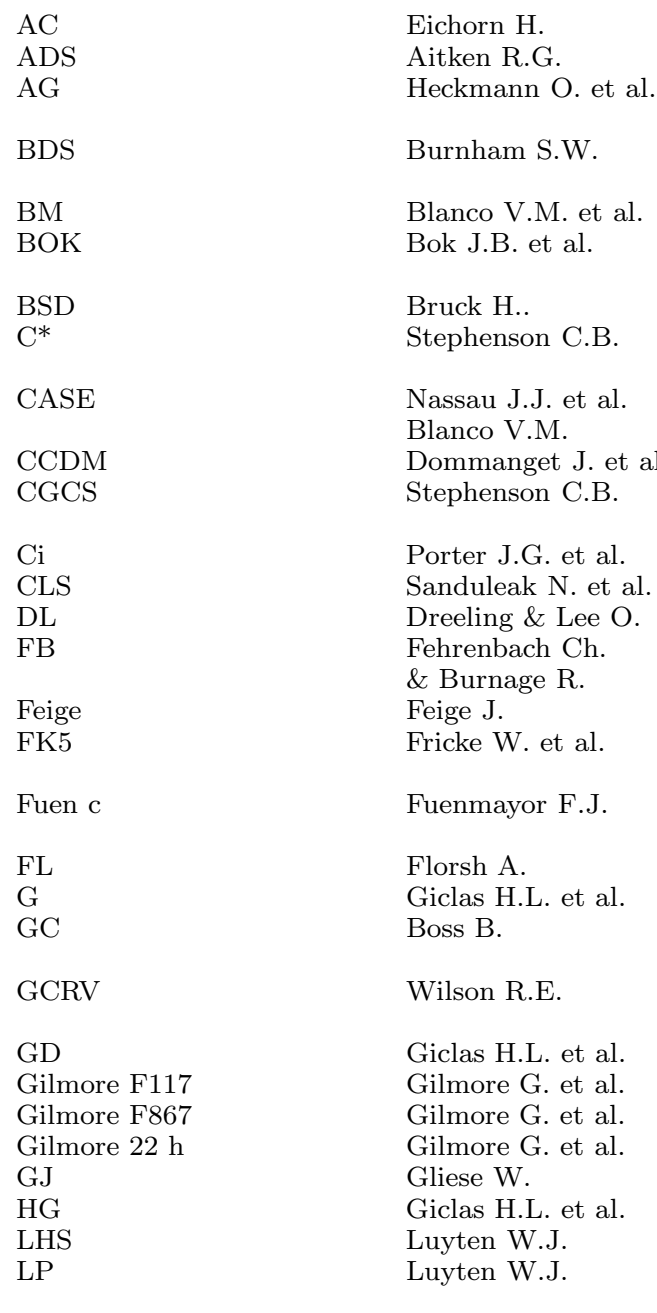

LS

LSS

LTT
MC

$\mathrm{McC}$

MO

NGP

NIKC

PLX

PMMR

PS

RM

SA

SAO

SMCOA

SNGP

VM

Volf

VVO

WEIS

WUD

$\mathrm{YZ}$
Astrographic Catalogue, 1974

New General Catalogue of Double Stars within $120^{\circ}$ of the North Pole, 1932

Astr. Gesellschaft Katalog. Star Catalogue of Positions and

Proper Motions North of $-2.5^{\circ}$ declination, 1975

A General Catalogue of Double Stars within $121^{\circ}$ of the

North Pole. Carnegie Inst. Washington Publ. No. 5, 1906

Bol. Obs. Tonantzinla Tacubaya 2, 17-1955

Stellar Distribution near the South Galactic Pole.

Mem. Mount Stromlo Obs. 16, 1964

Postdamer Obsev. Spectral Durchmusterung Post. Publ. 28, 1-1935

A general catalogue of Cool Carbon Stars,

Publ. Warner \& Swansey Obs. 1, 1, 1973

Carbon Stars ApJ 120, 129-1954, ApJ 125, 195-1957

Carbon Stars ApJ 127, 1958

Catalogue of Components of Double and Multiple Stars, 1994

A General Catalogue of Cool Galactic Carbon Stars

Publ. Warner \& Swansey Obs. 3, 53-1983

A Catalogue of Proper Motion Stars. Publ. Cincinnati Obs. 20, 1-1930

ApJS 66, 387-1988

Communication privée, 1978

A\&AS 49, 483-1982

A search for underluminous Stars. ApJ 128, 267-1958

Fifth Fundamental Catalogue. Veröff. Astron. Rechen-Inst.

Heidelberg 32, 1988

A Deep Near Infrared Obj-Prism Survey for Carbon Stars toward the Galactic Center and Anticenter. Rev. Mex. Astron. Astrofis. 6, 83-1981 Pub. Observ. Strasbourg, Vol. 2, 129, 1972

Lowell Proper Motion Survey. Lowell Obs. Bul. 89-165, 1959-1978

General Catalogue of 33342 Stars for the Epoch 1950. Carnegie Inst.

Washington Pub. No. 468, Vols. 1-5. Dudley Obs. Albany NY, 1937

General Catalogue of Stellar Radial Velocities, Carnegie Instit.

Washington Pub. No. 601, Mount Wilson Obs. Pasadena, California, 1953

A List of With Dwarf Suspects. Lowell Obs. Bul. 6-8, 1965-1978

MN 238, 225-1989

MN 213, 257-1985

Catalogue of Nearby Stars, Veröff. Astron. Rechen-Inst. Heidelberg 22, 1-1969

A Proper Motion Survey in the Hyades. Lowell Obs. Bul. 5, No. 118, 257-1962

Luyten Half-Second Catalogue, Univ. Minnesota, Minneapolis, 1979

Proper Motion Survey with the 48 inch Schmidt Luyten Palomar

Catalogue, 1963-1987

Luminous stars in the Northern Milky Way. Hamburger Sternwarte

Warney and Swasey Obs. Hamburg-Bergedorf, Vols. 1-6, 1959-1965

Stephenson C.B. et al. Luminous stars in the southern Milkey Way. Pub. Warner and Swasey

Obs. 1,1971

Luyten Two-Tenth Catalogue, Univ. Minnesota, Minneapolis, 1957

AJ 72, 1199, 1967

McCormick Observatory, ApJ 97, 381, 1943. ApJ 104, 234, 1946

ApJ 116, 117, 1952. AJ 61, 201, 1956. AJ 63, 211, 1958

AJ $82,474-1977$

AJ $67,37-1962$

Afz 26, 209-1987

Afz 29, 470-1988

General Catalogue of Trigonometric Stellar Parallaxes. Yale Obs.

New Haven, U.S.A., 1952

Catalogue of Late-Type Supergiants in the SMC. AAPS 53, 255-1983

Bol. Obs. Tonantzinla Tacubaya 4, 253-1968

AAPS 51, 277-1983

Ann. Harv. Coll. Obs. 101

SAO Catalogue (Smithsonian Institute Washington) DC, 1966

AJ 89, 1897-1984

AJ 93, 624-1987

AJ 94, 399-1941

Veröff. Sternw. Heidelberg 7, No. 10, 1919

Van Vleck Obs., parallaxes and proper motion. AJ 73-104, 1968-1992

Communication privée

AJ 86, 246-1981

Yale Photographic Catalogue, Trans. Astr. Obs. Yale Univ. Vols. 3-32, 1926-83 